\title{
Genetic characterization of red-colored heartwood genotypes of Chinese fir using simple sequence repeat (SSR) markers
}

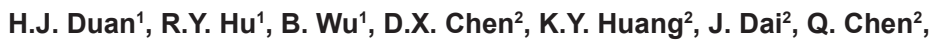 \\ Z.C. Wei ${ }^{3}$, S. Cao' ${ }^{1}$, Y.H. Sun ${ }^{1}$ and $Y$. $\mathrm{Li}^{1}$ \\ ${ }^{1}$ National Engineering Laboratory for Tree Breeding, \\ Key Laboratory of Genetics and Breeding in Forest Trees and Ornamental Plants, \\ Ministry of Education, College of Biological Sciences and Technology, \\ Beijing Forestry University, Beijing, China \\ ${ }^{2}$ Guangxi Province Forestry Research Institute, Nanning, Guangxi Province, China \\ ${ }^{3}$ State-Run Bei River Forest Farm, Rongshui, Guangxi Province, China \\ Corresponding author: Y.H. Sun / Y. Li \\ E-mail: sunyuhan@bjfu.edu.cn / yunli@bjfu.edu.cn
}

Genet. Mol. Res. 14 (4): 18552-18561 (2015)

Received July 28, 2015

Accepted October 7, 2015

Published December 28, 2015

DOI http://dx.doi.org/10.4238/2015.December.28.2

ABSTRACT. The present study investigated the genetic characterization of red-colored heartwood Chinese fir [Cunninghamia lanceolata (Lamb.) Hook.] in Guangxi using 21 simple sequence repeat (SSR) markers and analyzes of the genetic variation $(N=149)$ in samples obtained from five sites in Guangxi Province, China. The number of different alleles and the Shannon's information index per locus ranged from 3 to 12 and from 0.398 to 2.258 with average values of 6 and 1.211, respectively, indicating moderate levels of genetic diversity within this germplasm collection. The observed and expected heterozygosity ranged from 0.199 to 0.827 and from 0.198 to 0.878 with an average of 0.562 and 0.584 , respectively. Although, the mean fixation index was 0.044 , indicative of a low level of genetic differentiation among germplasms, analysis of molecular variance revealed considerable differentiation (99\%) within the 
samples. The neighbor-joining dendrogram revealed that the majority of red-colored Chinese fir genotypes were apparently not associated with their geographic origins. Further analysis by STRUCTURE showed that this Guangxi germplasm collection could be divided into three genetic groups comprising 76,37 , and 36 members, respectively; these were classified into mixed groups with no obvious population structure. These results were consistent with those of the cluster analysis. On the whole, our data provide a starting point for the management and conservation of the current Guangxi germplasm collection as well as for their efficient use in Chinese fir-breeding programs.

Key words: Red-colored heartwood; Chinese fir; Genetic diversity; Genetic differentiation; Molecular marker

\section{INTRODUCTION}

Chinese fir [Cunninghamia lanceolata (Lamb.) Hook] is an important indigenous tree species in China with many industrial applications due to its high growth rate, wood quality, and the versatility of its wood (Shi et al., 2010). It is widely cultivated in southern China. According to the multiple provenance testing results of 1976, a set of stands of Chinese fir were identified as elite provenance resources (Zheng et al., 2013). Of these, red-colored heartwood (a unique variety of Chinese fir) is particularly important because of its high wood quality and a higher ratio of hearts that improve the wood density and material properties, yielding commercial benefits and providing aesthetic value to solid wood utilization (Zhang, 2009).

Genetic variability is important for this tree species, especially for those varieties with high economic value for conservation and breeding. Abundant genetic variation allows the development of broad forest varieties adapted to multiple environmental conditions and enhances the genetic gain for several useful traits (Hoffmann and Sgrò, 2011; Matuszewski et al., 2015; Lopes et al., 2015). The biological factors inherent in a particular species, such as gene flow, divergence between populations, and reduction in diversity, can be elucidated based on genetic diversity and population structure, measured using molecular markers (Frankham et al., 2002). Previous studies on the Chinese fir have focused on characterizing genetic variation (Chung et al., 2009; Huang, 2010; Ou et al., 2014; Hao et al., 2014; Xu et al., 2014; Zheng et al., 2015), which could be used for germplasm conservation programs as well as for increasing our understanding of their biological characteristics (Eizirik, 1996). As appropriate evaluation of redcolored heartwood Chinese fir is required for understanding their genetic background, red-colored heartwood Chinese fir samples were collected from Guangxi province and conserved in the ex situ gene bank of Rongshui State-run Bei River Forest Farm (Guangxi, China). Understanding the genetic diversity of red-colored heartwood Chinese fir in Guangxi is important for effective utilization of its germplasm resources and for broadening the genetic base of this species to achieve continuous improvement.

Genetic diversity analysis requires suitable markers. Among the various molecular markers available for Chinese fir (You and Hong, 1998; Shen et al., 2011; Wen et al., 2013), simple sequence repeats (SSR) are the most popular because they are highly co-dominant, informative, and hypervariable (Ellis et al., 2007). Therefore, we used SSR markers to discern the DNA variation of this germplasm collection and to better understand the level of genetic variability as well as the 
relationship and population structure of this species distributed within the selected regions with the aim of enhancing the efficiency of Chinese fir-breeding programs.

\section{MATERIAL AND METHODS}

\section{Plant material and DNA extraction}

In the present study, 149 genetic resources of red-colored heartwood Chinese fir were analyzed using five samples collected from the Guangxi province. These genetic resources have been well-conserved in the ex situ gene bank of Rongshui State-run Bei River Forest Farm (Guangxi, China). Genomic DNA was extracted from mature leaves of the Chinese fir using a Plant Genomic DNA Kit (TIANGEN, Beijing, China). The quality and concentration of the extracted DNA was determined by electrophoresing the DNA samples on a $1 \%$ agarose gel.

\section{SSR analysis}

Genomic-SSR polymerase chain reaction (PCR) was performed in a $25-\mu \mathrm{L}$ reaction volume containing 1.0 $\mu \mathrm{L}$ genomic DNA (about $100 \mathrm{ng}$ ), $1.0 \mu \mathrm{L}$ forward $10 \mu \mathrm{M}$ primer, $1.0 \mu \mathrm{L} 10$ $\mu \mathrm{M}$ reverse primer, $12.5 \mu \mathrm{L} 2 \mathrm{X}$ Taq Plus PCR MasterMix (TIANGEN, Beijing, China), and $9.5 \mu \mathrm{L}$ double distilled water. The reaction was performed in the $\mathrm{T}_{100}{ }^{\mathrm{TM}}$ thermal cycler made by $\mathrm{BIO}$ RAD in the Key Laboratory of Genetics and Breeding in Forest Trees and Ornamental Plants with an initial denaturation at $94^{\circ} \mathrm{C}$ for $5 \mathrm{~min}$, followed by 35 cycles at $94^{\circ} \mathrm{C}$ for $30 \mathrm{~s}, 56 / 58 / 62^{\circ} \mathrm{C}$ (annealing temperature depending on the primer used in the reaction) for $30 \mathrm{~s}, 72^{\circ} \mathrm{C}$ for $30 \mathrm{~s}, 72^{\circ} \mathrm{C}$ (final extension) for $10 \mathrm{~min}$. Twenty-one microsatellite loci from the 35 samples investigated earlier (Wen et al., 2013) were selected for analysis (Table 1), based on the degree of polymorphism as well as the clarity and reproducibility of the amplified DNA fragments. The forward primer of each pair was labeled with a fluorescent dye (ROX, FAM, or HEX) during the synthesis. The PCR products were separated by capillary electrophoresis using an ABI3730xI DNA Analyzer (Applied Biosystems, Carlsbad, CA, USA). The amplicons were analyzed using Gene-Marker 2.2.0 software (SoftGenetics LLC, State College, PA, USA).

\section{Data analysis}

Convert 1.3.1 (Du et al., 2012) was used to convert microsatellite data to various formats for further analysis. Polymorphism of the SSR markers was evaluated by analyzing the genotypes from the five areas. Genetic diversity, including the number of alleles $\left(N_{\mathrm{A}}\right)$, effective number of alleles $\left(N_{E}\right)$, Shannon's Information Index $(\mathrm{I})$, observed heterozygosity $\left(H_{\mathrm{O}}\right)$, expected heterozygosity $\left(H_{\mathrm{E}}\right)$, Gene Flow $(\mathrm{Nm})$, and F-statistics calculations $\left(F_{\mathrm{IS}}, F_{\mathrm{IT}}\right.$, and $\left.F_{\mathrm{ST}}\right)$ were calculated using GenAIEx 6.5 software (Peakall and Smouse, 2006). Polymorphism information content (PIC) was calculated using PowerMarker v3.25 (Liu and Muse, 2005). AMOVA (analysis of molecular variance) was conducted using GenAIEx 6.5 combined with Microsoft Excel 2010 software (Peakall and Smouse, 2006). A neighbor-joining (NJ) tree was generated based on pairwise genetic distances among individuals using PowerMarker 3.25 (Liu and Muse, 2005). The program FigTree v. 1.3.1 (Rambaut, 2009) was employed to visualize and edit the resulting tree. 
Table 1. EST-SSR markers used in the study.

\begin{tabular}{|c|c|c|c|c|c|}
\hline Locus & Primer sequence $\left(5^{\prime}\right.$ - $\left.3^{\prime}\right)$ & Repeat motif & GenBank accession no & Anneal temperature $\left({ }^{\circ} \mathrm{C}\right)$ & Size Rang (bp) \\
\hline \multirow[t]{2}{*}{ SSR1 } & F: CAATCAGCCAAGTTGTACAGGC & (AT)8(AG)18 & AB757708 & 62 & $343-375$ \\
\hline & R: CATACCTTAGCAAAGCCCTCAGC & & AB757709 & & \\
\hline \multirow[t]{2}{*}{ SSR2 } & F: TGAAATTGCGTTGTACCGAAGG & $(\mathrm{GA}) 13$ & AB749554 & 56 & 273-297 \\
\hline & R: TAACGAGACGAGCGACAATCTCC & & AB749555 & & \\
\hline \multirow[t]{2}{*}{ SSR3 } & F: GATCCTCTGGTACTTGGTGCCC & (AT) 9 & AB749556 & 56 & $186-190$ \\
\hline & R: TGCAAAGTCATGTCATCTCTGGC & & AB749557 & & \\
\hline \multirow[t]{2}{*}{ SSR4 } & F: TGAATGGACTGCCACAAATTCC & (AG)11 & AB749550 & 56 & $288-298$ \\
\hline & R: TTCTTTGCAGGAAAGCCAACAAG & & AB749551 & & \\
\hline \multirow[t]{2}{*}{ SSR5 } & F: CTTAAGATAGCAGCGGGAATGG & (CT)11 & AB749562 & 56 & $258-279$ \\
\hline & R: CTTGCTCGATTTCTTGCATCTGG & & AB749563 & & \\
\hline \multirow[t]{2}{*}{ SSR6 } & F: AGAAATGTATACGGACCCTGCG & $(\mathrm{TA}) 9$ & AB749564 & 62 & $247-255$ \\
\hline & R: AAATCAAAGCACACGGTGAGAGC & & AB749565 & & \\
\hline \multirow[t]{2}{*}{ SSR7 } & F: AAGAGAAGAGGAGGAGGTCCAAG & $(\mathrm{AG}) 9$ & AB749570 & 56 & $269-283$ \\
\hline & R: CAGGAGCAGGTGCAGTAGCATTC & & AB749571 & & \\
\hline \multirow[t]{2}{*}{ SSR8 } & F: ATTATCCGAGGCAGATACGCAC & $(G G A) 10$ & AB749572 & 56 & $336-354$ \\
\hline & R: CTTCTCCGTATTTGATCCATCGC & & AB749573 & & \\
\hline \multirow[t]{2}{*}{ SSR9 } & F: GAGCCGTGAAGAACGAAGGTCTC & $(\mathrm{GAA}) 12$ & AB749574 & 56 & $260-281$ \\
\hline & R: ACGATCGGATTGTCTCAGAAACG & & AB749575 & & \\
\hline \multirow[t]{2}{*}{ SSR10 } & F: TGATCTTGGCATGTCAGTCTGG & (AT) 9 & AB749576 & 56 & $144-158$ \\
\hline & R: TGTCTGTCTGCCTGCAGTTATGC & & AB749577 & & \\
\hline \multirow{2}{*}{ SSR11 } & F: TCATCAGCCTCAGTTTGTACTTGC & (AT) 9 & AB749584 & 56 & $348-384$ \\
\hline & R: GCAATCATGGGCTCTCTGCAC & & AB749585 & & \\
\hline \multirow[t]{2}{*}{ SSR12 } & F: AATGCGACTTGCAAATTTCTGG & $(A G A) 10$ & AB749582 & 56 & $240-258$ \\
\hline & R: CGAATTCCTCAATCACTTGGCTG & & AB749583 & & \\
\hline \multirow[t]{2}{*}{ SSR13 } & F: GCGGCCATTTATATCATCTTC & $(\mathrm{GAA}) 9$ & AB749588 & 56 & $126-135$ \\
\hline & R: CACGCCTGTAATTCATCTCCGTC & & AB749589 & & \\
\hline \multirow[t]{2}{*}{ SSR14 } & F: GGTACTGCGAATCTTCAAATCC & $(\mathrm{TC}) 9$ & AB749580 & 56 & 293-297 \\
\hline & R: TGTTCAAGAAAGGAAGCAAACGG & & AB749581 & & \\
\hline \multirow[t]{2}{*}{ SSR15 } & F: TTTGGGACCTTATGGAGGTGGAG & $(G G A) 9$ & AB749602 & 56 & $153-159$ \\
\hline & R: AAACCACCAGGTTGAGAAGCAGC & & AB749603 & & \\
\hline \multirow[t]{2}{*}{ SSR16 } & F: TTTCGGCTCTCCGACTCCTTAAC & (CT)11 & AB749594 & 56 & $129-147$ \\
\hline & R: AGAATCGCGTCCAGAACACAGAG & & AB749595 & & \\
\hline \multirow[t]{2}{*}{ SSR17 } & F: TCCAGGAGTCTGTGAATCCGAAG & (CTG)9 & AB749600 & 56 & $223-238$ \\
\hline & R: CAGTACCAATTCAACCCAGCAGC & & AB749601 & & \\
\hline \multirow[t]{2}{*}{ SSR18 } & F: TCAAGAAGTTCCGCCATTGAGAG & (CTT)10 & AB749590 & 56 & $354-375$ \\
\hline & R: CCCATGAGGATTCAGAAACATGC & & AB749591 & & \\
\hline \multirow[t]{2}{*}{ SSR19 } & F: TTAATGGTGCAAGGTGGAATTGG & $(\mathrm{GAA}) 10$ & AB749592 & 56 & $280-286$ \\
\hline & R: TTGAATCCCACTGATCACACTGC & & AB749593 & & \\
\hline \multirow[t]{2}{*}{ SSR20 } & F: CTGTTTGTACATTGGCCTCGAC & (СТC)9 & AB749596 & 58 & $136-148$ \\
\hline & R: TCACAAACCACTGTGCTGGAATG & & AB749597 & & \\
\hline \multirow[t]{2}{*}{ SSR21 } & F: GTTGGGATGTCATCAAGATTGG & (TC) 11 & AB749598 & 58 & $226-270$ \\
\hline & R: TGAAAGAGGCGGAAATTGGTAGG & & AB749599 & & \\
\hline
\end{tabular}

The population structure was analyzed using the Bayesian model-based clustering approach with STRUCTURE v2.3.1 (Pritchard et al., 2000). A burn-in period was used; the number of Markov chain Monte Carlo (MCMC) reps after burn-in was 100,000, with a 100,000-run length, with 10 replicate runs for each analysis, and subpopulations $(K)$ ranging from 1 to 10 . The approach to maximum likelihood with Ln probability of data \pm SD [LnP (D); Pritchard et al., 2000] and the calculation of $\Delta \mathrm{K}$ by Evanno et al. (2005) was employed to determine the most probable value for K using Structure Harvester (Earl and Vonholdt, 2012).

\section{RESULTS}

\section{Assessment of genetic diversity using SSR markers}

A total of 149 red-colored heartwood Chinese fir accessions from Guangxi province, 
including Sirong (SR:89 accessions), Yongle (YL:36 accessions), Xiadong (X:13 accessions), Gaoling (G:5 accessions), and a set of Gandong (GD:6 accessions), were analyzed using 21 SSR markers. The genetic profiles detected 122 alleles ranging from 3 to 12 per locus, with an average of 6 (Table 2). The mean $N_{E}$ was 3.422 , ranging from 1.272 to 8.895 . Shannon's information index (I) ranged from 0.398 to 2.258 , with an average of 1.211 . The $H_{\mathrm{O}}$ in the total sample was 0.562 , which was lower than the $\mathrm{H}_{\mathrm{E}}$, at $10 \mathrm{SSR}$ loci, in accordance with the mean fixation index $\left(F_{1 \mathrm{~S}}=\right.$ $0.014 ; \mathrm{P}<0.05)$. A moderate inbreeding coefficient $(0.056)$ using the $F_{\mathrm{IT}}$ and an $F_{\mathrm{ST}}$ value of 0.044 across all loci suggested genetic subdivision, indicative of low genetic differentiation among the sites. With an average of 6.968 , the values of gene flow $(\mathrm{Nm})$ ranged from 3.456 to 19.750. The majority of loci conformed to Hardy-Weinberg equilibrium (HWE). Furthermore, mean PIC loci was 0.75 , ranging from 0.42 to 0.97 (Table 2), which was reasonably informative. AMOVA revealed a low $(1 \%)$ but significant $(P<0.01)$ variation among samples, and $99 \%$ variation was attributed to differences within samples (Table 3 ).

Table 2. Summary statistics of genetic variation in Chinese fir accessions included in this study using SSR markers.

\begin{tabular}{lrlllllrrrr}
\hline locus & $N_{\mathrm{A}}$ & $N_{\mathrm{E}}$ & $\mathrm{I}$ & $H_{\mathrm{O}}$ & $H_{\mathrm{E}}$ & $F_{\text {IS }}$ & $F_{\text {IT }}$ & $F_{\text {ST }}$ & $N_{\mathrm{M}}$ & PIC \\
\hline SSR1 & 12 & 8.895 & 2.258 & 0.494 & 0.878 & 0.438 & 0.467 & 0.051 & 4.609 & 0.97 \\
SSR2 & 10 & 7.037 & 2.013 & 0.781 & 0.838 & 0.069 & 0.107 & 0.042 & 5.749 & 0.97 \\
SSR3 & 3 & 1.365 & 0.478 & 0.290 & 0.263 & -0.105 & -0.091 & 0.013 & 19.75 & 0.42 \\
SSR4 & 8 & 4.300 & 1.669 & 0.811 & 0.762 & -0.064 & -0.002 & 0.059 & 3.989 & 0.92 \\
SSR5 & 6 & 2.616 & 1.206 & 0.589 & 0.606 & 0.028 & 0.070 & 0.043 & 5.520 & 0.82 \\
SSR6 & 5 & 2.406 & 1.088 & 0.515 & 0.537 & 0.043 & 0.107 & 0.067 & 3.456 & 0.82 \\
SSR7 & 5 & 3.328 & 1.345 & 0.758 & 0.692 & -0.095 & -0.022 & 0.067 & 3.504 & 0.85 \\
SSR8 & 5 & 2.240 & 0.980 & 0.426 & 0.519 & 0.180 & 0.231 & 0.062 & 3.798 & 0.72 \\
SSR9 & 7 & 4.690 & 1.680 & 0.827 & 0.786 & -0.053 & -0.024 & 0.028 & 8.724 & 0.93 \\
SSR10 & 4 & 2.476 & 1.009 & 0.561 & 0.579 & 0.031 & 0.089 & 0.060 & 3.918 & 0.78 \\
SSR11 & 10 & 7.329 & 2.056 & 0.644 & 0.845 & 0.238 & 0.278 & 0.053 & 4.476 & 0.97 \\
SSR12 & 3 & 1.272 & 0.398 & 0.199 & 0.198 & -0.005 & 0.047 & 0.052 & 4.553 & 0.41 \\
SSR13 & 4 & 2.288 & 0.931 & 0.583 & 0.546 & -0.069 & -0.014 & 0.052 & 4.586 & 0.70 \\
SSR14 & 4 & 2.341 & 0.965 & 0.552 & 0.569 & 0.031 & 0.047 & 0.017 & 14.349 & 0.71 \\
SSR15 & 5 & 2.089 & 0.968 & 0.532 & 0.501 & -0.062 & -0.015 & 0.044 & 5.391 & 0.72 \\
SSR16 & 6 & 2.184 & 1.022 & 0.622 & 0.535 & -0.163 & -0.097 & 0.057 & 4.133 & 0.75 \\
SSR17 & 4 & 1.373 & 0.553 & 0.264 & 0.267 & 0.013 & 0.041 & 0.029 & 8.504 & 0.45 \\
SSR18 & 5 & 3.556 & 1.421 & 0.724 & 0.715 & -0.013 & 0.004 & 0.016 & 15.150 & 0.89 \\
SSR19 & 3 & 1.595 & 0.641 & 0.401 & 0.362 & -0.108 & -0.071 & 0.033 & 7.262 & 0.53 \\
SSR20 & 3 & 1.720 & 0.701 & 0.476 & 0.415 & -0.148 & -0.121 & 0.024 & 10.286 & 0.54 \\
SSR21 & 10 & 6.763 & 2.038 & 0.756 & 0.841 & 0.101 & 0.147 & 0.051 & 4.623 & 0.97 \\
Mean & 6 & 3.422 & 1.211 & 0.562 & 0.584 & 0.014 & 0.056 & 0.044 & 6.968 & 0.75 \\
C.V & 44.91 & 65.63 & 45.93 & 0.03 & 34.98 & & & & & \\
Total & 122 & & - & - & & & & \\
\hline
\end{tabular}

$N_{\mathrm{A}}$ : Number of Different Alleles; $N_{\mathrm{E}}$ : Number of Effective Alleles; I: Shannon's Information Index; $H_{\mathrm{O}}$ : Observed Heterozygosity; $H_{\mathrm{E}}$ : Expected Heterozygosity; $F_{\mathrm{IS}}$ : inbreeding coefficient; $F_{\mathrm{IT}}$ : over inbreeding coefficient; $F_{\mathrm{ST}}$ : fixation index; $N_{\mathrm{M}}$ : Gene Flow; PIC: polymorphic information content.

Table 3. Analysis of molecular variance (AMOVA).

\begin{tabular}{|c|c|c|c|c|c|c|}
\hline Source & $\mathrm{df}$ & SS & MS & Est. Var. & $\%$ & $P$ value \\
\hline Among the populations from Guangxi & 4 & 63.045 & 15.761 & 0.072 & 1 & $<0.01$ \\
\hline Within a population from Guangxi & 144 & 2046.224 & 14.210 & 14.210 & 99 & $<0.01$ \\
\hline Total & 148 & 2109.268 & & 14.282 & 100 & \\
\hline
\end{tabular}




\section{Genetic differentiation among five regions}

Further analyses of $N_{\mathrm{A}}, N_{\mathrm{E}}, \mathrm{I}, H_{\mathrm{O}}$, and $H_{\mathrm{E}}$ (Table 4) were effected to estimate the variations among the samples obtained from five different regions. The highest degree of genetic diversity was observed in $\operatorname{SR}\left(N_{\mathrm{A}}=8.333, N_{\mathrm{E}}=4.077, \mathrm{I}=1.388, H_{\mathrm{O}}=0.576\right.$, and $\left.H_{\mathrm{E}}=0.624\right)$ and $\mathrm{Y}\left(N_{\mathrm{A}}=7.571, N_{\mathrm{E}}=\right.$ $4.009, \mathrm{I}=1.392, H_{\mathrm{O}}=0.595$, and $\left.H_{\mathrm{E}}=0.627\right)$, whereas the diversity was lowest in $\mathrm{GD}\left(N_{\mathrm{A}}=3.810, N_{\mathrm{E}}=\right.$ 2.707, I = 0.992, $H_{\mathrm{O}}=0.484$, and $\left.H_{\mathrm{E}}=0.521\right)$ and $\mathrm{G}\left(N_{\mathrm{A}}=3.952, N_{\mathrm{E}}=3.109, \mathrm{I}=1.085, H_{\mathrm{O}}=0.610\right.$, and $\left.H_{\mathrm{E}}=0.568\right)$. The $H_{\mathrm{O}}$ value was significantly lower than the $H_{\mathrm{E}}$ value in all the populations, except $\mathrm{G}$. This result is consistent with the high value of the fixation index. The value of $N_{A}$ and the number of private alleles in SR was far higher than those in other samples, possibly because of the large disparities in sample size (Santos, 2015). The pairwise genetic differentiation values $\left(F_{\mathrm{ST}}\right)$ calculated for the samples from five regions showed genetic differentiation between each region (Table 5), with the values ranging from -0.00642 to 0.02862 . Among the samples from five populations, those from GD population had higher $F_{\mathrm{ST}}$ value, possibly due to a history of genetic drift. Rousset's genetic distance values $\left[F_{\mathrm{ST}} /\right.$ $\left.\left(1-F_{\mathrm{ST}}\right)\right]$ (Rousset, 1997) indicated that the most closely related regions were $\mathrm{G}$ and SR, whereas the largest Rousset's distance $(0.0295)$ was between $G$ and GD.

Table 4. Genetic diversity parameters for the Guangxi Chinese fir samples.

\begin{tabular}{lrcccccrrr}
\hline Pop & $\mathrm{N}$ & $\mathrm{SN}$ & $N_{\mathrm{A}}$ & $N_{\mathrm{E}}$ & $\mathrm{I}$ & $H_{\mathrm{O}}$ & $H_{\mathrm{E}}$ & $\mathrm{F}$ & Private alleles \\
\hline SR & 89 & $1-89$ & 8.333 & 4.077 & 1.388 & 0.576 & 0.624 & 0.059 & 23 \\
$\mathrm{GD}$ & 6 & $90-95$ & 3.810 & 2.707 & 0.992 & 0.484 & 0.521 & 0.021 & 1 \\
X & 13 & $96-108$ & 5.286 & 3.209 & 1.195 & 0.546 & 0.578 & 0.021 & 3 \\
Y & 36 & $109-144$ & 7.571 & 4.009 & 1.392 & 0.595 & 0.627 & 0.037 & 13 \\
G & 5 & $145-149$ & 3.952 & 3.109 & 1.085 & 0.610 & 0.568 & -0.095 & 1 \\
\hline
\end{tabular}

SN: Serial Number.

\begin{tabular}{|c|c|c|c|c|c|}
\hline & $S R$ & GD & $x$ & $\mathrm{Y}$ & G \\
\hline SR & 0.00000 & & & & \\
\hline GD & 0.02043 & 0.00000 & & & \\
\hline$x$ & 0.00381 & 0.01170 & 0.00000 & & \\
\hline Y & 0.00261 & 0.01420 & 0.00834 & 0.00000 & \\
\hline G & -0.00642 & 0.02862 & 0.00111 & -0.00086 & 0.00000 \\
\hline
\end{tabular}

\section{Cluster analysis}

An unrooted neighbor-joining (NJ) tree (Figure 1) was constructed based on the pairwise genetic distances using PowerMarker V3.25 (Liu and Muse, 2005). Broadly, 149 Chinese fir germplasms could be divided into three main clusters ( $A, B$, and $C$ ), among which $B$ and $C$ were further divided into two subclusters (B1 and B2; C1 and C2). Genotypes from SR were distributed in all the clusters, with maximum percentage in cluster B; $63.89 \%$ of genotypes from $Y$ belonged to subcluster B2, and the others to cluster $\mathrm{C}$, of which only one belonged to subcluster $\mathrm{C} 1$. Only one genotype originated from $\mathrm{G}$ belonged to cluster $\mathrm{C}$, and the other four belonged to cluster B. Genotypes from $\mathrm{X}$ belonged to cluster B and C and did not cluster into the same class. Thus, we redefined the relationship between the resources not related to their natural regional distributions. Of these subclusters, subcluster B2 contained the maximum $(\mathrm{N}=82$ ) members from divergent populations. 


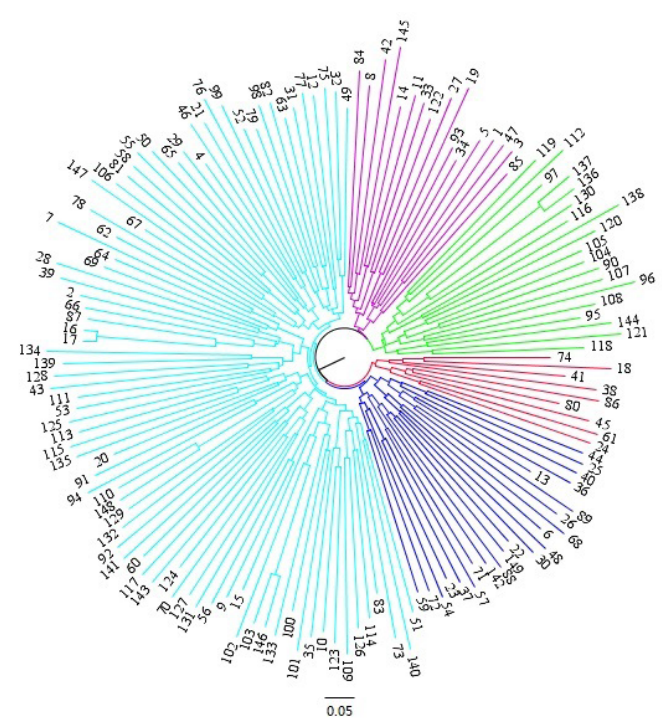

Figure 1. Neighbor joining analysis of the genetic similarity among 149 red-colored Chinese fir genotypes. Red represents group A, deep blue represents group B1, Cambridge blue represents group B2, purple represents group $\mathrm{C} 1$, and green represents group $\mathrm{C} 2$.

\section{Population genetic structure}

To further explore the genetic structure of these germplasm resources, population structure analysis was performed. When $\mathrm{K}=3, \Delta \mathrm{K}$ showed a clear peak, suggesting that there were three major clusters in the above provenance (Figure 2 and Figure 3). Except the individuals of the GD population, members of all others populations were present in each cluster. About $51 \%$ of individuals belonged to Cluster I, which consisted of 47 and $72 \%$ of individuals of populations SR and Y, respectively. Cluster II and III consisted of 37 and 36 individuals, respectively, from all populations. The three clusters were dominated by different gene pools but showed similar ancestry that caused non-conspicuous genetic differentiation from one another.

A

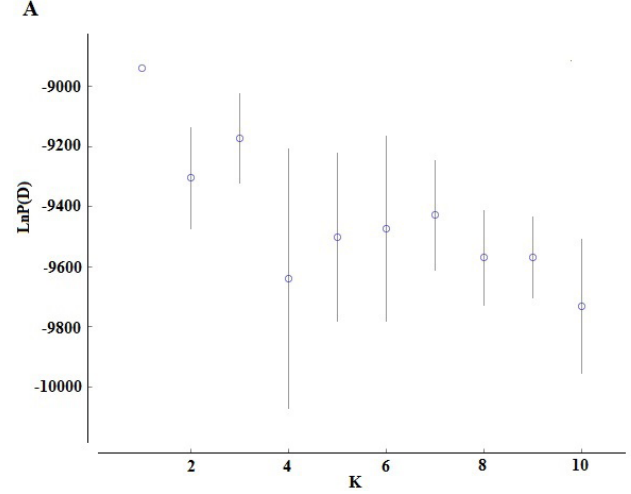

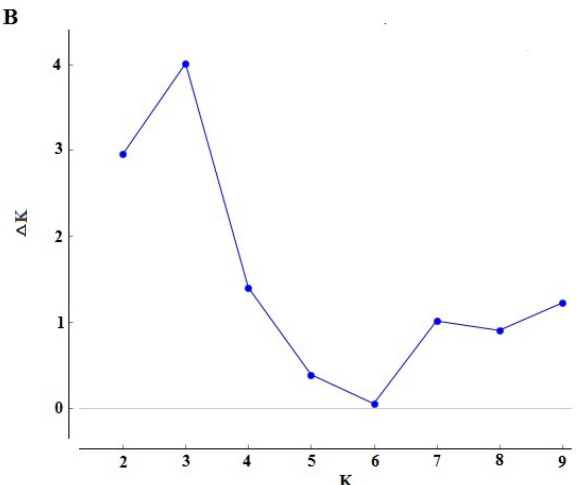

Figure 2. Plot of the $L n P(D) \pm S D$ and delta $K(D K)$. A. The mean of $L n P(D)$ was based on 10 repetitions for each $K$ value. B. Plot of $\Delta \mathrm{K}$ according to $\mathrm{K}$. 


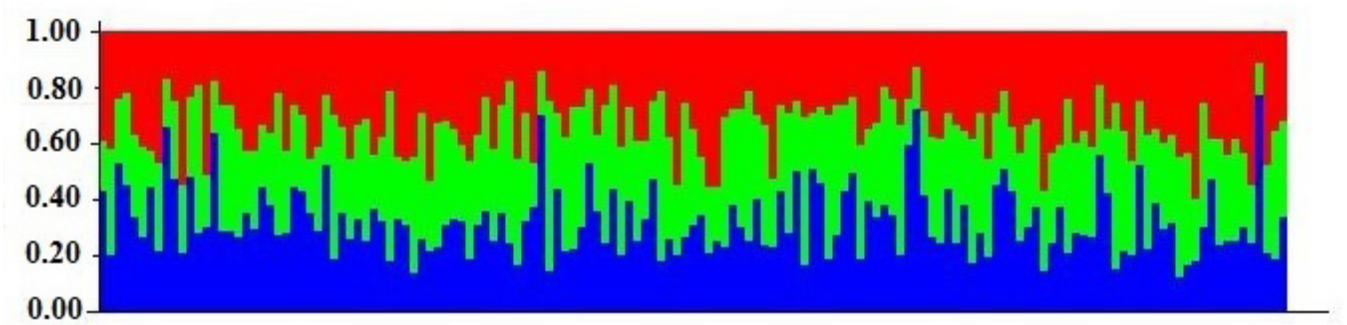

Figure 3. Genetic structure of the Guangxi Chinese fir germplasm collection. Graphic representation of the genetic structure of the Guangxi Chinese fir germplasm collection $(N=149)$ for $K=3$. Each individual is represented by a vertical bar divided into segments representing the estimated member ship proportion in the three cluster inferred with STRUCTURE.

\section{DISCUSSION}

Genetic evaluation is important for germplasm utilization in tree breeding. In the present study, 21 SSR primer combinations yielded 122 alleles with a mean of 6 per locus in 149 genotypes of red-colored heartwood Chinese fir. This value was equal to the $N_{\mathrm{A}}(6)$ obtained from 16 Chinese fir individuals based on 28 microsatellite markers (Wen et al., 2013). Among these, SSR6 had the highest diversity, with 12 alleles. Such a significant number of alleles may be related to the codominant SSR markers (Du et al., 2012) and was higher than the values that have been reported for other populations of Chinese fir identified using SSR markers (Ou et al., 2014; Xu et al., 2014) as well as the obtained PIC (0.42-0.97) and Shannon's diversity index values (0.398-2.258). Long life, outcrossing, and wind-pollination are prevalent in this species (Zhang and Lin, 1990), with only a narrow geographical range showing similar genetic backgrounds. This may explain the considerable level of genetic diversity observed in this species. The mean value of $F_{\mathrm{ST}}$ was 0.044 , which is indicative of a low level of population differentiation (Wright, 1978). It was confirmed by AMOVA, where only $1 \%$ of the total molecular variance was attributable to the interpopulation level (Table 3).

Heterozygosity is an important indicator of gene diversity (Slatkin and Barton, 1989). In this study, the mean $H_{\mathrm{O}}$ and $H_{\mathrm{E}}$ levels of heterozygosity were 0.562 and 0.584 , respectively. Lower values were reported for other populations of Chinese fir using SSR markers (Ou et al., 2014; Xu et al., 2014). $H_{\mathrm{E}}(0.584)$ obtained using SSR markers was also higher for some conifers, including Pinus bungeana ( $H_{\mathrm{E}}$ : 0.249; Zhao et al., 2014), Pinus massoniana $\left(H_{\mathrm{E}}\right.$ : 0.536; Bai et al., 2014), and Pinus dabeshanensis ( $H_{\mathrm{E}}$ : 0.458; Xiang et al., 2015). However, it is lower than that of Abies chensiensis $\left(H_{\mathrm{E}}: 0.803\right.$; Li et al., 2012), and Austrocedrus chilensis $\left(H_{\mathrm{E}}\right.$ : 0.865 ; Colabella et al., 2014). Further studies have indicated that the genetic diversity of this germplasm resource is moderate. In this study, $H_{\mathrm{O}}$ obtained was higher than the $H_{\mathrm{E}}$ at $11 \mathrm{SSR}$ loci, which is indicative of an excess of heterozygosity at these loci. Hybridization of multiple related species at different geographic locations, over a long period of time, may have caused the excess heterozygotes across the entire natural distribution range (Li et al., 1997). However, the $H_{\mathrm{O}}$ was lower than the $H_{\mathrm{E}}$ for the other 10 SSR loci and for the mean value of all 21 sites. Fewer heterozygotes were observed at the population level, except in $\mathrm{G}$ population. Similar findings have been reported in many other tree species (Veron et al., 2005; Hadziabdic et al., 2012; Wang et al., 2014). A process of inbreeding in these trees, such as partial selfing (Veron et al., 2005), effect of biparental breeding because of limited pollen dispersal among relatives (Hadziabdic et al., 2012), and the result of half-sibling mating occurring over a small geographical area, may have caused the heterozygote deficiency 
(Wang et al., 2014). Self-pollination found in Chinese fir (Zhang and Lin, 1990) effecting low levels of inbreeding may result in the deficiency of heterozygotes in red Chinese fir, observed in our study. Further studies are required to decipher the precise cause of the heterozygote deficiency.

In the neighbor-joining ( $\mathrm{NJ}$ ) dendrogram, it was observed that the majority of red-colored Chinese fir genotypes were not obviously related to the geographic origins. The genetic structure results further supported that the majority of genotypes were classified into a mixed group without ascertaining their geographic origins. This may be because germplasms collected from closer geographic distances showed non-conspicuous genetic differentiation or because they have a history of genetic drift. However, the moderate levels of genetic diversity but low degrees of genetic differentiation of the tested germplasms may be because of the limited number of SSR primers, not evenly distributed throughout the genome. More SSR primers or other markers, such as single-nucleotide polymorphisms (SNPs), may be applicable (Las et al., 2014) for effective genetic characterization and population structure analysis of Chinese fir. Genetic variations in red-colored heartwood Chinese fir have been analyzed in a variety of species (Zhang, 2009); however, further experiments are required to study Chinese fir genetic diversity. Further understanding of population structure is required to perform association analyses. In future, more unique shape control genes and diverse genotypes should be selected to improve the exploitation of available Chinese fir resources and augment the breeding process based on morphological traits and genetic diversity. However, based on the genetic relationships of the tested germplasms, it is possible to improve the germplasm resources and select appropriate materials for breeding.

\section{Conflicts of interest}

The authors declare no conflicts of interest.

\section{ACKNOWLEDGMENTS}

Research supported by the National ' 863 ' Project in the 12th Five-Year Plan in China (\#2011AA100203), the Key Projects of the State Forestry Administration (\#2012-06), and the Forestry Science and Technology Projects of Guangxi province (\#2014-01).

\section{REFERENCES}

Bai TD, Xu LA, Xu M and Wang ZR (2014). Characterization of masson pine (Pinus massoniana Lamb.) microsatellite DNA by 454 genome shotgun sequencing. Tree Genet. Genomes 10: 429-437.

Chung JD, Chien CT, Nigh G Nd Ying CC (2009). Genetic Variation in Growth Curve Parameters of Konishii fir (Cunninghamia lanceolata (LAMB.) HOOK. var. konishii). Silvae Genet. 58: 1-2.

Colabella F, Gallo LA, Moreno AC and Marchelli P (2014). Extensive pollen flow in a natural fragmented population of Patagonian cypress Austrocedrus chilensis. Tree Genet. Genomes 10: 1519-1529.

Du Q, Wang B, Wei Z, Zhang D, et al. (2012). Genetic diversity and population structure of Chinese white poplar (Populus tomentosa) revealed by SSR markers. J. Hered. 103: 853-862.

Earl DA and vonHoldt BM (2012). STRUCTURE HARVESTER: a website and program for visualizing STRUCTURE output and implementing the Evanno method. Conserv. Genet. Resour. 4: 359-361.

Eizirík E (1996). Ecologia molecular, genética da conservação eo conceito de unidades evolutivamente significativas. Braz. J. Genet. 19: 23-29.

Ellis JR and Burke JM (2007). EST-SSRs as a resource for population genetic analyses. Heredity (Edinb.) 99: 125-132.

Evanno G, Regnaut S and Goudet J (2005). Detecting the number of clusters of individuals using the software STRUCTURE: a simulation study. Mol. Ecol. 14: 2611-2620.

Frankham R, Briscoe DA and Ballou JD (2002). Introduction to conservation genetics. Cambridge University Press, Cambridge. Hadziabdic D, Wang X, Wadl PA, Rinehart TA, et al. (2012). Genetic diversity of flowering dogwood in the Great Smoky 
Mountains National Park. Tree Genet. Genomes 8: 855-871.

Hao BB, Zou F, Hu SL and Xu GB (2014). Genetic Diversity of Chinese Fir Provenances Using ISSR Markers. Guangxi For. Sci. 1: 004.

Hoffmann AA and Sgrò CM (2011). Climate change and evolutionary adaptation. Nature 470: 479-485.

Huang $\mathrm{HH}$ (2010). Investigation and genetic diversity analysis of the king of lanceolata in Fujian Provenances, Fujian Agriculture and Forestry University.

Las Casas G, Scollo F, Distefano G, Continella A, et al. (2014). Molecular characterization of olive (Olea europaea L.) Sicilian cultivars using SSR markers. Biochem. Syst. Ecol. 57: 15-19.

Li KY, Huang MR, and Wang MX (1997). Study on the origin of Populus tomentosa Carr. using the RAPD method. Acta Phytotax. Sin. 35: 24-31.

Li WM, Li SF and Li B (2012). Genetic diversity in natural populations of Abies chensiensis based on nuclear simple sequence repeat markers. Chin. Bull. Bot. 47: 413-421.

Liu K and Muse SV (2005). PowerMarker: an integrated analysis environment for genetic marker analysis. Bioinformatics 21: 2128-2129.

Lopes MS, El-Basyoni I, Baenziger PS, Singh S, et al. (2015). Exploiting genetic diversity from landraces in wheat breeding for adaptation to climate change. J. Exp. Bot. erv122.

Matuszewski S, Hermisson J and Kopp M (2015). Catch me if you can: Adaptation from standing genetic variation to a moving phenotypic optimum. Genetics: 115: 178574.

Ou YL, Chen JH, Zheng RH, Xu Y, et al. (2014). Genetic diversity among the germplasm collections of the Chinese fir in 1st breeding population upon SSR markers. J. Nanj. For. Univ. Nat. Sci. Ed. 38: 21-26.

Peakall R and Smouse PE (2006). GENALEX 6: genetic analysis in Excel. Population genetic software for teaching and research. Mol. Ecol. Notes 6: 288-295.

Pritchard JK, Stephens M and Donnelly P (2000). Inference of population structure using multilocus genotype data. Genetics 155: 945-959.

Rambaut A (2009). FigTree, ver.1.3.1.[Online]. Available at [http://tree.bio.ed.ac. uk/software/figtree/] Accessed September 2014.

Santos RR, Cavallari MM, Pimenta MA, Abreu AG, et al. (2015). Population genetic structure of Attalea vitrivir Zona (Arecaceae) in fragmented areas of southeast Brazil. Genet. Mol. Res. 14: 6472-6481.

Shen A, Li H, Wang K, Ding H, et al. (2011). Sequence characterized amplified region (SCAR) markers-based rapid molecular typing and identification of Cunninghamia lanceolata. Afr. J. Biotechnol. 10: 19066-19074.

Shi J, Zhen Y and Zheng RH (2010). Proteome profiling of early seed development in Cunninghamia lanceolata (Lamb.) Hook. J. Exp. Bot. 61: 2367-2381.

Slatkin M and Barton NH (1989). A comparison of three indirect methods for estimating average levels of gene flow. Evolution 43: 1349-1368.

Veron V, Caron H and Degen B (2005). Gene flow and mating system of the tropical tree Sextonia rubra. Silvae Genet. 54: 275-280.

Wang Z, Kang M, Liu H, Gao J, et al. (2014). High-level genetic diversity and complex population structure of Siberian apricot (Prunus sibirica L.) in China as revealed by nuclear SSR markers. PLoS One 9: e87381.

Wen Y, Ueno S, Han W and Tsumura Y (2013). Development and characterization of 28 polymorphic EST-SSR markers for Cunninghamia lanceolata (Taxodiaceae) based on transcriptome sequences. Silvae Genet. 62: 137-141.

Wright S (1978). Evolution and genetics of populations variability within and among populations. University of Chicago Press, Chicago.

Xiang XY, Zhang ZX, Duan RY, Zhang XP, et al. (2015). Genetic diversity and structure of Pinus dabeshanensis revealed by expressed sequence tag-simple sequence repeat (EST-SSR) markers. Biochem. Syst. Ecol. 61: 70-77.

Xu Y, Chen JH, Zhao YQ, Wang Y, et al. (2014). Variation of EST-SSR molecular markers among provenances of Chinese fir. J. Nanj. For. Univ. Nat. Sci. Ed. 38: 1-8.

You Y and Hong JS (1998). The application of the genetic variation of Chinese fir provenance using RAPD markers. Sci. Silvae $\operatorname{Sin}$. 34: 32-38.

Zhang X (2009). Genetic variation on the trait of Red-Colored Heart wood of Cunninghamia lanceolata (Lamb.) Hook. Master's thesis, Nanjing Forestry University, Nanjing.

Zhang ZW and Lin P (1990). The ecology characteristics of pollen research of Chinese Fir. Forest Sci. 26: 410-418.

Zhao HH, Zheng YQ, Li B, Zhang CH, et al. (2014). Genetic diversity analysis of Pinus bungeana natural populations with ESTSSR markers. Forest Res. 27: 474-480.

Zheng HQ, Hu DH, Wang RH, Wei RP, et al. (2013). Plus tree resource survey and genebank construction for Cunninghamia lanceolata. J. South. Forest. Coll. 1: 22-26.

Zheng HQ, Duan HJ, Hu DH, Wei RP, et al. (2015). Sequence-related amplified polymorphism primer screening on Chinese fir (Cunninghamia lanceolata (Lamb.) Hook). J. For. Res. 26: 101-106. 